\title{
Wideband H-Slot Antenna Fed by Substrate Integrated Gap Waveguide for mmWave Arrays
}

\author{
Ryosuke Kon ${ }^{1}$, Wai Yan Yong ${ }^{2 *}$, and Andrés Alayón Glazunov ${ }^{2,3}$ \\ ${ }^{1}$ School of Engineering, Tohoku University, Japan \\ ${ }^{2}$ Department of Electrical Engineering, University of Twente, 7522NB, Enschede, The Netherlands \\ ${ }^{3}$ Department of Electrical Engineering, Chalmers University of Technology, Gothenburg, Sweden \\ * Corresponding author, email: w.y.yongwaiyan@utwente.nl
}

\begin{abstract}
This paper proposes a wideband slot antenna design based on the substrate integrated gap waveguide technology for millimetre wave applications. The proposed antenna is realized using the $\mathrm{H}$-shaped slot $(\mathrm{H}$-slot) to improve impedance bandwdith. Simulation results show that the proposed antenna has an impedance bandwidth at $S_{11} \leq-15$ dB from $24-29.5$ GHz resulting in a $20.5 \%$ fractional bandwidth. Hence, the designed slot antenna can potentially be used as the radiating element of an array antenna for fixed-beam point-to-point applications.
\end{abstract}

Keywords-Gap waveguide, millimeter-wave, substrate integrated gap waveguide

\section{INTRODUCTION}

Microstrip lines have been commonly used in the design of millimetre wave (mmWave) antennas because of their convenient integration with the RF circuitry, and also due to their cost-effective manufacturing [1], [2]. A drawback is that the dielectric losses at mmWave frequencies are high. An alternative is to use metal hollow waveguides as the transmission line since low loss array antennas can be designed at the mmWave bands [3]. However, a good electrical contact is required for the satisfactory performance of a multi-layer antenna resulting in higher manufacturing costs [3]. To address the aforementioned problem, the gap waveguide (GW) has been proposed as an effective transmission line solution for mmWave antennas [4]. The use of the fully metallic GW keep losses at a comparable level with the conventional hollow waveguides, while circumventing the strict electrical contact requirements [4]. However, in practice, it is difficult to maintain a constant air gap height between the layers, which may have a major impact on the performance of the GW [4]. The substrate integrated gap waveguide (SIGW) has been proposed in order to overcome this constant air gap problem by filling it with a substrate [5]. In this paper, we propose an $\mathrm{H}$-shaped slot antenna (henceforth, the H-slot antenna) based on the SIGW technology for mmWave application. The proposed antenna is fed by a T-junction, which design is based on the SIGW technology. The simulated performance of the proposed antenna provides a $S_{11} \leq-15 \mathrm{~dB}$ impedance bandwidth of $20.5 \%$ from $24-29.5 \mathrm{GHz}$.

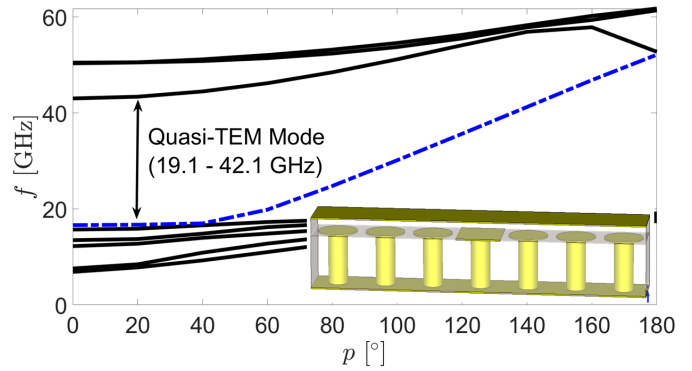

Fig. 1. Dispersion diagram of the designed SIGW, where $f$ is the frequency and $p$ is the phase in degree.

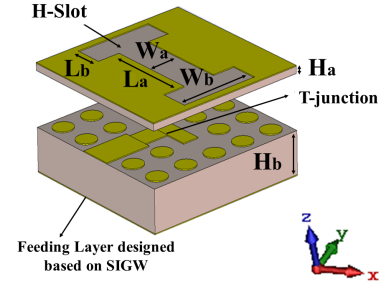

(a)

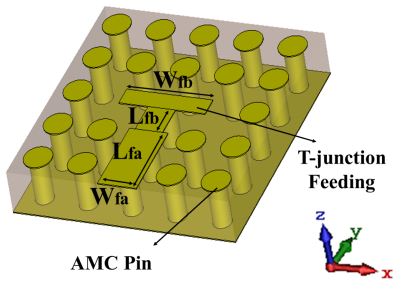

(b)
Fig. 2. Explored view of the proposed antenna (a) H-shaped slot antenna fed by SIGW and (b) T-junction feeding designed based on SIGW.

\section{SIGW H-SLOT ANTENNA DESIGN AND RESULTS}

In the design of the SIGW antenna it is important to ensure that the artificial magnetic conductor (AMC) pins provides a stop-band over the desired operating frequency [4]. Fig. 1 shows the dispersion diagram of the designed SIGW structure shown in the figure insert. The SIGW consists of a periodic AMC pin realized as the combination of a mushroom patch and metal vias, and the guiding ridge is made of a copper patch and metal vias. As can be seen from the dispersion diagram, only a single propagating mode is possible from $19.1-42.1 \mathrm{GHz}$. In this band, the quasiTEM mode is propagating in the substrate gap between the ridge and the upper copper patch [5]. The completed design of the SIGW is used to realize a T-junction to fed to the $\mathrm{H}$-slot antenna as shows in Fig. 2. As can be seen from Fig. 2, the proposed antenna comprises two layers: the top 
TABLE I. Dimensions of the proposed slot antenna fed by SIGW.

\begin{tabular}{cccccc}
\hline Parameters & $W_{a}$ & $\mathrm{~W}_{b}$ & $L_{a}$ & $L b$ & $H_{a}$ \\
\hline Dimension $(\mathbf{m m})$ & 2.3 & 4.1 & 4.5 & 1.9 & 0.508 \\
\hline Parameters & $H_{b}$ & $W_{f a}$ & $W_{f b}$ & $L_{f a}$ & $L_{f b}$ \\
\hline Dimension $(\mathbf{m m})$ & 1.575 & 1.8 & 3.5 & 3 & 1.2 \\
\hline
\end{tabular}

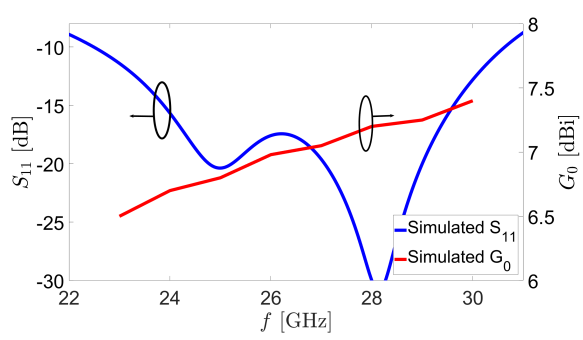

Fig. 3. Simulated input reflection coefficient, $S_{11}$ and the realized gain $G_{0}$ of the proposed slot antenna fed by SIGW.

radiating layer and the bottom SIGW feeding layer. The air gap between the radiating and feeding layers is filled with the substrate. The width and length of the unit cell antenna is designed to be around $0.95 \lambda_{0}$, where $\lambda_{0}$ is the free-space wavelength at $31 \mathrm{GHz}$. The optimized parameters of the proposed antenna are tabulated in Table I. The H-slot is proposed as the radiating slot to improve the bandwidth performance as compared to the conventional radiating slot. The operating bandwidth of the conventional radiating slot is primarily determined by the length of the slot. The H-slot, in turn, operates like multiple ridge slots, allowing the slot to shift the cut-off frequency to a wider frequency band of operation.

Fig. 3 shows the simulated input reflection coefficient $S_{11}$ and the realized gain $G_{0}$ of the proposed H-slot antenna fed by the SIGW. As can be seen from Fig. 3, the proposed antenna provides an $-15 \mathrm{~dB}$ impedance bandwidth of $20.5 \%$ from $24-29.5 \mathrm{GHz}$. In addition, the antenna provides a simulated realized gain above $6.5 \mathrm{dBi}$. The radiation pattern at 24,26 , and $28 \mathrm{GHz}$ at the E- (XOZ) and the H-(YOZ) planes are shown in Fig. 4. The radiation patterns are nearly identical over the desired operating frequency. The $3 d B$ beamwidth of the proposed slot antenna is more than $85^{\circ}$ with a change of less than $8^{\circ}$ over the operating band for both $\mathrm{E}$ - and H-planes. In addition, the relative cross-polarization (X-pol) level is less than $-10 \mathrm{~dB}$ for both the E- and the $\mathrm{H}$-planes over the operating frequencies. Compared to the solutions proposed in [1] and [2], our design provides a much wider impedance bandwidth of around $20.5 \%$ covering the potential frequencies for mmWave $5 \mathrm{G}$. In addition, the conventional PCB manufacturing technique can be used to produce the SIGW as demonstrated in [2].

\section{CONCLUSION}

A wideband high-gain slot antenna fed by a substrate integrated gap waveguide (SIGW) has been designed. The combined use of the H-slot and the SIGW T-junction feeding

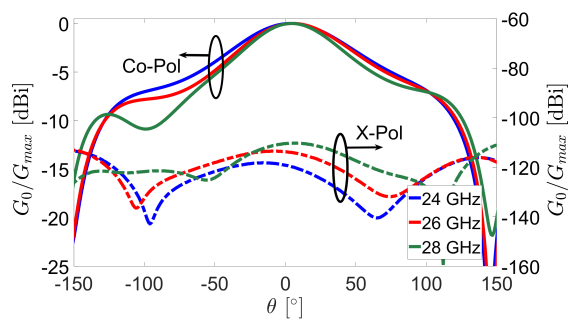

(a)

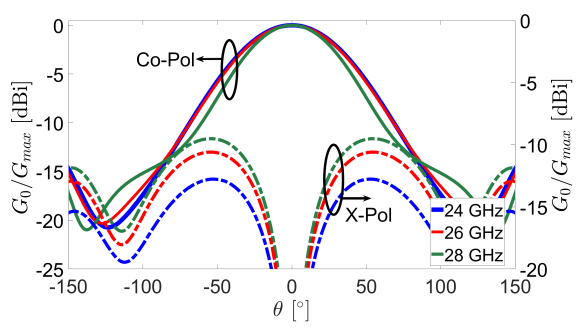

(b)

Fig. 4. Simulated radiation pattern of the proposed H-slot antenna fed by SIGW for (a) E-plane and (b) H-plane at 24, 26 and $28 \mathrm{GHz}$, respectively. $G_{0} / G_{\max }$ denotes the normalized gain and $\theta$ is the polar angle in degrees.

line resulted in a $20.5 \%$ impedance bandwidth from $24-29.5$ $\mathrm{GHz}$ at the $-15 \mathrm{~dB}$ reflection coefficient level. The proposed antenna have a realized gain approximately from $6.5-7.5$ $\mathrm{dBi}$. Hence, the proposed antenna design is a good radiating element candidate for mmWave array antenna applications. Future work will focus to optimized and extend the unit cell slot antenna into a larger array antenna configuration with low side lobe and low cross-polarization level for all E-, D- and H-planes to realize a high-gain fixed-beam antenna which is suitable for point-to-point communication.

\section{ACKNOWLEDGMENTS}

This project has received funding from the EU H2020 research and innovation program under the Marie SklodowskaCurie grant agreement No. 766231-WAVECOMBE-H2020MSCA-ITN-2017. Ryosuke Kon was supported by the Gyomu Super Japan Dream Foundation during his six-month exchange at the University of Twente.

\section{REFERENCES}

[1] W. Y. Yong and A. A. Glazunov, "High Gain, Wideband Grid Array Antenna for $28 \mathrm{GHz} 5 \mathrm{G}$ Base Station," in 2019 13th European Conference on Antennas and Propagation (EuCAP). IEEE, 2019, pp. 1-5.

[2] P. A. Dzagbletey and Y.-B. Jung, "Stacked microstrip linear array for millimeter-wave $5 \mathrm{~g}$ baseband communication," IEEE Antennas and Wireless Propagation Letters, vol. 17, no. 5, pp. 780-783, 2018.

[3] T. Tomura, Y. Miura, and M. Zhang, "A $45^{\circ}$ Linearly Polarized HollowWaveguide Corporate-Feed Slot Array Antenna in the 60-GHz Band,' IEEE Trans. Antennas Propag., vol. 60, no. 8, pp. 3640-3646, 2012.

[4] P.-s. Kildal, E. Alfonso, and E. Rajo-iglesias, "Local MetamaterialBased Waveguides in Gaps Between Parallel Metal Plates," IEEE Antennas and Wireless Propagation Letters, vol. 8, pp. 84-87, 2009.

[5] J. Zhang, X. Zhang, and A. A. Kishk, "Design of substrate integrated gap waveguide and their transitions to microstrip line, for millimeterwave applications," IEEE Access, vol. 7, pp. 154 268-154 276, 2019. 